This is an electronic reprint of the original article. This reprint may differ from the original in pagination and typographic detail.

Author(s): Neittaanmäki, N.; Salmivuori, M.; Pölönen, Ilkka; Jeskanen, L.; Ranki, A.; Saksela, O.; Snellman, E.; Grönroos, M.

Title: Hyperspectral imaging in detecting dermal invasion in lentigo maligna melanoma

Year: $\quad 2017$

Version:

Please cite the original version:

Neittaanmäki, N., Salmivuori, M., Pölönen, I., Jeskanen, L., Ranki, A., Saksela, O., Snellman, E., \& Grönroos, M. (2017). Hyperspectral imaging in detecting dermal invasion in lentigo maligna melanoma. British Journal of Dermatology, 177(6), 1742 1744. https://doi.org/10.1111/bjd.15267

All material supplied via JYX is protected by copyright and other intellectual property rights, and duplication or sale of all or part of any of the repository collections is not permitted, except that material may be duplicated by you for your research use or educational purposes in electronic or print form. You must obtain permission for any other use. Electronic or print copies may not be offered, whether for sale or otherwise to anyone who is not an authorised user. 
Received Date : 23-May-2016

Revised Date : 06-Dec-2016

Accepted Date : 20-Dec-2016

Article type : Research Letter

\section{Hyperspectral imaging in detecting dermal invasion in lentigo maligna}

\section{melanoma}

N. Neittaanmäki ${ }^{1}$, M. Salmivuori ${ }^{2}$, I. Pölönen ${ }^{3}$, L. Jeskanen ${ }^{4}$, A. Ranki ${ }^{4}$, O. Saksela ${ }^{4}$, E. Snellman ${ }^{5}$, M. Grönroos ${ }^{2}$

(1) Department of Clinical Pathology, Sahlgrenska University hospital, Göteborg, Sweden

(2) Department of Dermatology and Allergology, Päijät-Häme Social and Health Care Group, Lahti, Finland

(3) Department of Mathematical Information Technology, University of Jyväskylä, Finland

(4) Department of Dermatology and Allergology, Helsinki University and Helsinki University Hospital, Finland

(5) Department of Dermatology, Tampere University and Tampere University Hospital, Finland

Corresponding author: Noora Neittaanmäki, MD, PhD,

Department of Clinical Pathology, Sahlgrenska University Hospital, Gula Stråket 8, 41345 Göteborg, Sweden

E-mail: noora.neittaanmaki@fimnet.fi

Funding: This study was funded by the Novo Nordisk Foundation's Novo PreSeed Grant, by the Instrumentarium Foundation and by the Finnish Cancer foundation.

Conflict of interest: The authors declare no conflicts of interest.

This article has been accepted for publication and undergone full peer review but has not been through the copyediting, typesetting, pagination and proofreading process, which may lead to differences between this version and the Version of Record. Please cite this article as doi: $10.1111 /$ bjd.15267

This article is protected by copyright. All rights reserved. 
What's already known about this topic? Recently, various non-invasive imaging tools have been introduced for detection of skin cancers. Hyperspectral imaging is a novel promising technology, which combines spectroscopy and skin imaging and enables analysis of large skin areas at once.

What does this study add? This pilot study shows that hyperspectral imaging can be used in preoperative staging of lentigo maligna melanomas

\section{TO THE EDITOR}

Invasive lentigo maligna melanoma (LMM) and its in situ precursor lentigo maligna (LM) are the most common melanoma types on the head and neck areas ${ }^{1}$. Clinically unsuspected invasion is revealed histologically in 5$52 \%$ of LMs ${ }^{2}$. Thus, correct early diagnoses are crucial for determining accurate resection margins and the discussion of sentinel lymph node examination ${ }^{3}$.

Hyperspectral imaging is based on the detection of spectral differences reflecting a tissue's biological properties and can be used for detecting skin malignancies ${ }^{4,5}$. The hyperspectral imaging is rapid and spectral data can be obtained from images with a large imaging field. We determined how accurately a hyperspectral imaging system (HIS) detected LMM invasion.

A total of 31 patients, with 32 lesions, with histologically confirmed LM or LMM, participated in the study. The patients' mean age was 77 years (range 56-97), 16 were female and 15 were male. Four patients had skin photo-type I, 17 had type II and 10 had type III. Fifteen patients had earlier had keratinocyte (pre)malignancies. None of the patients had received immunosuppressant, cytostatics, biological agents or radiotherapy to the studied areas. Two patients had previously received phototherapy.

Of the $32 \mathrm{LM} / \mathrm{LMM}$ lesions, 10 were histologically defined as LMMs (eight on the face or scalp, two on the trunk) with Breslow thicknesses between 0.4-1.6 mm (mean $0.85 \mathrm{~mm}$ ). The remaining 22 lesions were histologically LMs (20 on the face or scalp, two on the trunk).

All lesions were evaluated with dermatoscopy (Dermlite ${ }^{\circledR}$ 3GenCA, USA) and photographed (Canon Ixus 115 HS, 12.1 megapixel). Hyperspectral images were taken in vivo using a HIS camera prototype (VTT FPI VISVNIR Spectral Camera), based on a Fabry-Perot interferometer. Within seconds HIS captures the diffuse reflectance of visible and near-infrared light $\left(500-900 \mathrm{~nm}\right.$ ), with a $12 \mathrm{~cm}^{2}$ field of view (spatial resolution 6400 pixels $/ \mathrm{cm}^{2}$, pixel $=125 \mu \mathrm{m}$ ) and imaging depth of approx. $2 \mathrm{~mm}$. In acquired hyperspectral images every pixel 
represents a diffuse reflectance spectrum formed from the mixing of spectra from the different materials in the image. With mathematical modelling this spectrum can be separated into the constituent spectra from these different materials. These "pure" spectra that contribute to the mixed spectrum are called 'endmember' spectra, Fig 1. End-members can further be used to create abundance images showing localization of these spectra in the imaged area, Fig 2-3. The detailed description of the technique has been reported elsewhere ${ }^{4,5}$.

For the first four patients, the lesions were excised with 5mm-margins immediately after imaging. In the consequent cases, punch-biopsies were taken from the suspected invasion sites, and thereafter excised entirely using a $5 \mathrm{~mm}$-margin for LM and a $10 \mathrm{~mm}$-margin for LMM. The specimens were fixed in $4 \%$ formalin, embedded in paraffin, sectioned in 3-5 mm intervals and stained with hematoxylin-eosin and if needed with MART1 immunohistochemistry. The samples were evaluated by an experienced dermatopathologist (L.J.) blinded to the HIS outcome.

In abundance images, in situ LMs were seen as homogenous white areas while in the case of LMMs the abundance maps showed a dark hole on the site of invasion, and the rest of lesion was seen as a homogenous white area as in LM. These areas, which represented the LMM invasion, were seen as clear white areas in separate abundance maps, Fig 2. This finding was seen in 9/10 LMMs (true positives). HIS did not detect the invasion in one of the 10 LMMs, where the Breslow thickness was $0.5 \mathrm{~mm}$ (false negative). In 19/22 LMs HIS did not indicate any sites of invasion (true negatives), Fig 3, whereas in 3/22 cases invasions were suggested, but not detected histologically (false positives). Thus, HIS achieved a positive predictive value of $75 \%$, a negative predictive value of $95 \%$, a sensitivity of $90 \%$ and a specificity of $86.3 \%$.

Diffuse reflectance records both light absorption and scattering, and thus provides information on skins morphology and chromophore content. Abundance images represent the localization of each characteristic diffuse reflectance spectra (end-member) in the imaged area. In this study the abundance images showed clear differences between LM and LMM. Most likely, absorption of the skin chromophores (like melanin) at different skin levels explains most of the differences between the spectra.

Many techniques have been developed for melanoma diagnostics. Of these, dermatoscopy and reflectance confocal microscopy (RCM) are widely used ${ }^{6}$. Both techniques are excellent in giving the diagnosis, but imprecise in determining the thickness and early invasion. Multispectral imaging devices have also been introduced ${ }^{7-10}$. Multispectral devices use multiple (4-15) wide non-continuous bands of wavelengths resulting in discrete spectral images ${ }^{8,9}$, while HIS uses continuous narrow wavebands delivered from a tunable filter. 
Different tumor types can be identified by their different spectra ${ }^{11-13}$. Scanning and microscopic hyperspectral imagers have been assessed for detecting skin cancers ${ }^{11-13} \cdot{ }^{13}$ The HIS method used in this study to differentiate the in situ and invasive melanoma from each other, showed much promise in guiding practitioners to obtain more targeted diagnostic biopsies. HIS also proved to be useful for improving the detection and staging of LMM. HIS could also be developed further to measure the thickness of LMM non-invasively prior to surgery, which would save time and resources.

A limitation in our study was its small sample size. A larger multicenter study is warranted to confirm our results. Although the analyzing method is objective, clinicians still need to interpret the HIS images, which, however, needs less expertise than the interpretation of near-histological RCM images. The quality of the HIS images may be affected by artefacts caused by crusts, lesion thickness and differences in the skin color. The HIS is not yet in commercial production. A future goal is to image a large set of various skin tumors for the development of a classification algorithm for non-invasive tumor diagnostics based on spectral data.

A future prospective could be to combine different imaging techniques (dermatoscopy/RCM) and HIS for identification of invasive tumors.

To conclude, HIS is a promising tool to detect basal membrane invasion in LMM, and thus to separate in situ and invasive LMs for more accurate pre-surgical diagnostics.

\section{CONFLICT OF INTEREST}

The authors declare no conflicts of interest.

\section{ACKNOWLEDGEMENT}

This study was funded by the Novo Nordisk Foundation's Novo PreSeed Grant and by the Instrumentarium Foundation and by the Finnish Cancer foundation.

\section{REFERENCES}

1 Bosbous MW, Dzwierzynski WW, Neuburg M. Lentigo maligna: diagnosis and treatment. Clin Plast Surg $2010 ; 37: 35-46$.

2 McGuire LK, Disa JJ, Lee EH, et al. Melanoma of the lentigo maligna subtype: diagnostic challenges and current treatment paradigms. Plastic \& Reconstructive Surgery 2012; 129:288e-99e. 
3 Hieken TJ, Hernandez-Irizarry R, Boll JM, Jones Coleman JE. Accuracy of diagnostic biopsy for cutaneous melanoma: implications for surgical oncologists. International Journal of Surgical Oncology Print 2013; 2013:196493.

4 Neittaanmaki-Perttu N, Gronroos M, Tani T, et al. Detecting field cancerization using a hyperspectral imaging system. Lasers in Surgery \& Medicine 2013; 45:410-7.

5 Neittaanmaki-Perttu N, Gronroos M, Jeskanen L, et al. Delineating margins of lentigo maligna using a hyperspectral imaging system. Acta Derm Venereol 2015; 95:549-52.

6 Smith L, Macneil S. State of the art in non-invasive imaging of cutaneous melanoma. Skin Research \& Technology 2011; 17:257-69.

7 Hauschild A, Chen SC, Weichenthal M, et al. To excise or not: impact of MelaFind on German dermatologists' decisions to biopsy atypical lesions. Journal der Deutschen Dermatologischen Gesellschaft 2014; 12:606-14.

8 Glud M, Gniadecki R, Drzewiecki KT. Spectrophotometric intracutaneous analysis versus dermoscopy for the diagnosis of pigmented skin lesions: prospective, double-blind study in a secondary reference centre. Melanoma Res 2009; 19:176-9.

9 Sgouros D, Lallas A, Julian Y, et al. Assessment of SIAscopy in the triage of suspicious skin tumours. Skin Research \& Technology 2014; 20:440-4.

10 Monheit G, Cognetta AB, Ferris L, et al. The performance of MelaFind: a prospective multicenter study. Arch Dermatol 2011; 147:188-94.

11 Nagaoka T, Nakamura A, Okutani H, et al. A possible melanoma discrimination index based on hyperspectral data: a pilot study. Skin Research \& Technology 2012; 18:301-10.

12 Gaudi S, Meyer R, Ranka J, et al. Hyperspectral imaging of melanocytic lesions. Am J Dermatopathol 2014; 36:131-6.

13 Garcia-Uribe A, Zou J, Duvic M, et al. In vivo diagnosis of melanoma and nonmelanoma skin cancer using oblique incidence diffuse reflectance spectrometry. Cancer Res 2012; 72:2738-45.

\section{FIGURE LEGENDS}

Fig 1. Unsupervised mathematical modeling (linear signal un-mixing) was used to separate end-members i.e. characteristic spectra of LM, invasive LMM and healthy skin. These spectra were further used to create abundance images i.e. localization maps of these characteristic spectra in the imaged skin area (Figures 2-3).

Fig 2. Clinical images, histological images and hyperspectral abundance images representing LMM (true positive) a) Photograph of LMM b) Dermatoscopic image of LMM c) Hyperspectral abundance map representing the healthy skin around the lesion (white) d) Hyperspectral abundance map representing the noninvasive part of the LMM-lesion (white) e) Hyperspectral abundance map representing the dermal invasion in 
LMM seen in a separate abundance map, the red arrow points to the invasion area f) Haematoxylin and eosin staining of LMM (Breslow $1.6 \mathrm{~mm}$ ) g) Immunohistochemistry for MART1 in LMM (Breslow $1.6 \mathrm{~mm}$ )

Fig 3. Clinical images, histopathological images and hyperspectral abundance images representing in situ LM (true negative) a) Photograph of LM b) Dermatoscopic image of LM c) Hyperspectral abundance map representing the healthy skin around a lesion (white) d) Hyperspectral abundance map representing the LMlesion (white), no suspected invasion was seen e) Haematoxylin and eosin staining of LM

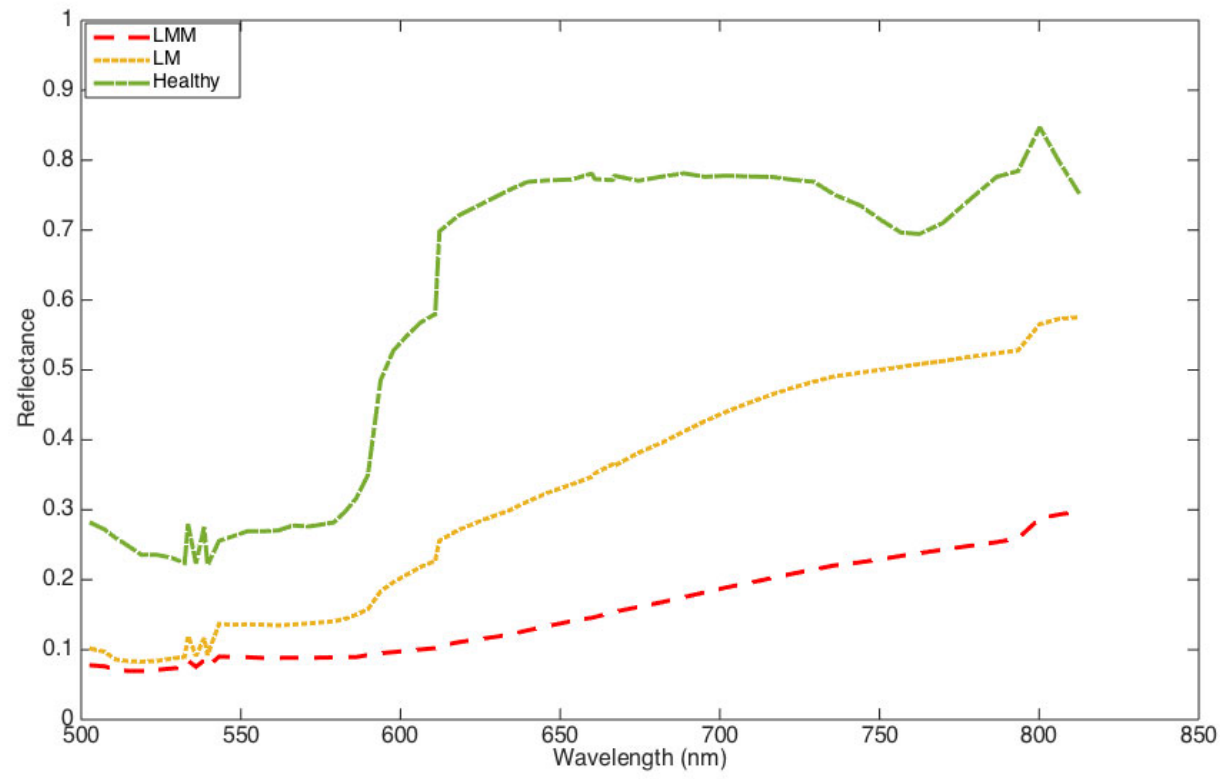

This article is protected by copyright. All rights reserved. 

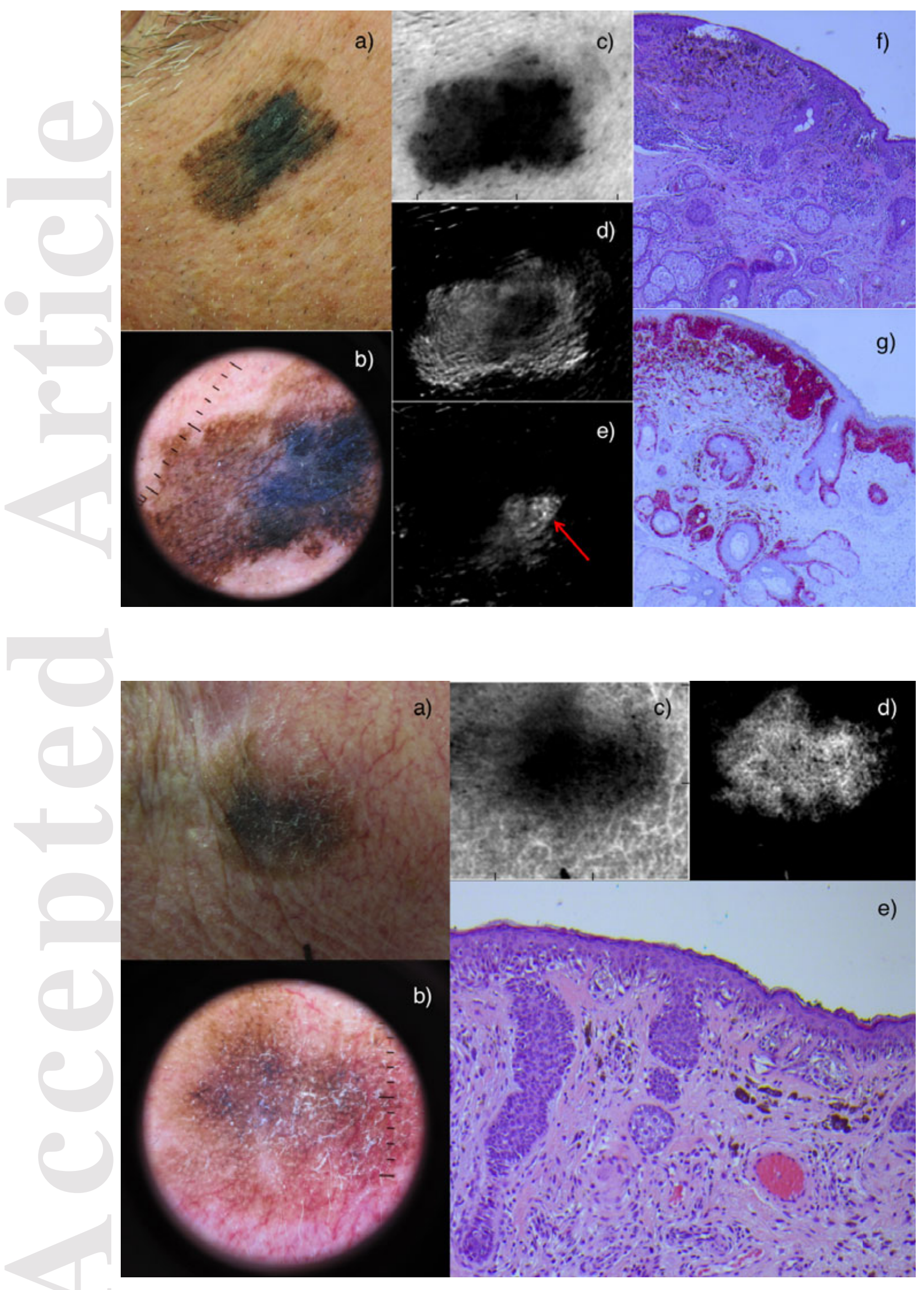

This article is protected by copyright. All rights reserved. 